\title{
Transformation of CSD When Crystal Shape Changes with Crystal Size into CLD from FBRM by Using Monte Carlo Analysis
}

\author{
Joi Unno, Izumi Hirasawa \\ Department of Applied Chemistry, School of Science and Engineering, Waseda University, Tokyo, Japan \\ Email: j.unno@fuji.waseda.jp
}

How to cite this paper: Unno, J. and Hirasawa, I. (2017) Transformation of CSD When Crystal Shape Changes with Crystal Size into CLD from FBRM by Using Monte Carlo Analysis. Advances in Chemical Engineering and Science, 7, 91-107. https://doi.org/10.4236/aces.2017.72008

Received: December 15, 2016

Accepted: February 6, 2017

Published: February 9, 2017

Copyright (c) 2017 by authors and Scientific Research Publishing Inc. This work is licensed under the Creative Commons Attribution International License (CC BY 4.0).

http://creativecommons.org/licenses/by/4.0/ c) (i) Open Access

\begin{abstract}
In manufacturing process, it is necessary to measure change in CSD (Crystal Size Distribution) with time accurately because CSD is one of the most important indices that evaluate quality of products. FBRM (Focused Beam Reflectance Measurement) can measure CLD (Chord Length Distribution) in line, but CLD is different from CSD because of principle of FBRM. However, if CSD is determined beforehand, CLD can be calculated from the CSD with statistical method. First, when crystal shape is defined from the characteristic crystal size, the matrix of each crystal shape which transforms CSD into CLD in a uniform manner is calculated with Monte Carlo analysis. Characteristic crystal size is added to the variables defining chord length in order to avoid complex integrals and apply the change in crystal shape with characteristic crystal size to the transforming matrix. Secondly, CSD and CLD are actually measured in suspension of acetaminophen in ethanol and suspension of Larginine in water to demonstrate the validity of 2 matrices. Lastly, these matrices are multiplied by some simple CSD models to test the properties of these matrices and demonstrate the utility of this transformation.
\end{abstract}

\section{Keywords}

Focused Beam Reflectance Measurement (FBRM), Chord Length

Distribution (CLD), Crystal Size Distribution (CSD),

Monte Carlo Analysis, Characteristic Crystal Size

\section{Introduction}

Inmanufacturing process of crystal, powder, or granule products, it is necessary to measure change in CSD (crystal size distribution) or PSD (particle size distribution) with time accurately because CSD is one of the most important indices 
that evaluate quality of products [1].

FBRM (focused beam reflectance measurement) can measure CLD (chord length distribution) in line, but it is well known that CLD is different from CSD because of principle of FBRM [2] [3]. In FBRM, focused beam from probe enters suspension in vessel, and chord length is measured based on detection time of backscattered light when beam runs cylindrically at high speed. Back scattered light results from beam which hits a crystal, but the chord length can differ from the crystal size because the beam doesn't necessarily scan the crystal along the characteristic crystal size. However, if CSD is determined beforehand, CLD can be calculated from the CSD with statistical method [4].

In this paper, first, when crystal shape was defined from the characteristic crystal size, the matrix of each crystal shape which transforms CSD into CLD in a uniform manner was calculated with Monte Carlo analysis. Secondly, CSD and CLD were actually measured in suspension of acetaminophen (AAP) in ethanol and suspension of $L$-arginine (Arg) in water to demonstrate the validity of 2 matrices. Lastly, these matrices were multiplied by some simple CSD models to test the properties of these matrices and demonstrate the utility of this transformation.

Because this transformation is simply represented by a matrix, it is easy to apply the matrix to inverse transformation and this method is assumed to contribute significantly to in-line measurement of CSD. In some of previous studies [2] [3], discretizing was used to solve complex integral problems. In this paper, by using Monte Carlo analysis instead of discretization, the transforming matrix can be calculated quickly and accurately. Translation, which is one of the variables defining chord length, was made to exist within variable range and weighting was performed for each range in many of previous studies [2] [3]. In this paper, translation is made to exist within fixed sufficient range in order to avoid weightings in CLD calculation process. In addition, characteristic crystal size is added to the variables defining chord length, which have been composed of rotation angles around 3 axes and a translation [2] [3] [4], in order to avoid complex integrals and apply the change in crystal shape with characteristic crystal size to the transforming matrix.

\section{Theory}

\subsection{Principle of Chord Length Measurement with FBRM}

Particle Track G400, which can measure CLD in line based on FBRM, was used in this paper. Focused beam from probe enters suspension in vessel, and chord length is measured within a range of 1 to $1000 \mu \mathrm{m}$ based on detection time of backscattered light when beam runs cylindrically at the speed of $2 \mathrm{~m} / \mathrm{sec}$. The concept of FBRM is shown in Figure 1.

In Figure 1, the cylinder along which the beam runs has a large diameter in comparison to crystals within the measuring range, and so the beam path can be regarded as a straight line. 


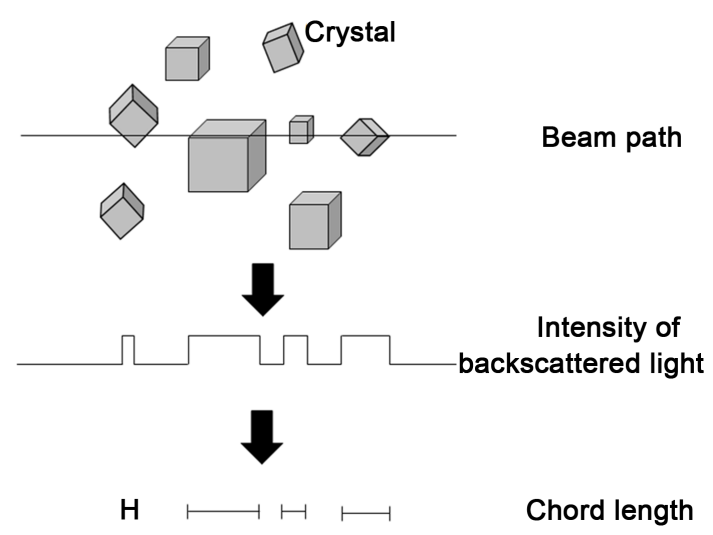

Figure 1. FBRM method of measurement.

As seen from Figure 1, because the parts of crystals where chord lengths are measured differ from one another, CLD differs from CSD.

\subsection{Function Which Determines Chord Length}

First, it is considered that crystal shape $\boldsymbol{P}$ is defined only from vertex coordinates and that the vertex coordinates are mapping of characteristic crystal size $L_{\mathrm{C} s}$. At this time, Equation (1) is established.

$$
\boldsymbol{P}\left(L_{\mathrm{CS}}\right)=\left(\boldsymbol{p}_{1}, \boldsymbol{p}_{2}, \cdots\right)=\left(\begin{array}{ccc}
x_{1} & x_{2} & \cdots \\
y_{1} & y_{2} & \cdots \\
z_{1} & z_{3} & \cdots
\end{array}\right)
$$

where $\boldsymbol{p}_{i}$ is the $\boldsymbol{i}$ th position vector of vertex coordinate and $\boldsymbol{P}$ is $3 \times m$ matrix in which $m$ position vectors of vertex coordinates defining crystal shape are placed in $m$ columns. $x$ Axis is defined as the beam scanning direction, $z$ axis as the beam traveling direction, and $y$ axis as the other direction. Due to calculation, one of the 2 points which are the most distant from each other of all the vertex coordinates is sited at the origin. This model can be used when crystals are regarded as polyhedra. Then, a domain of $L_{\mathrm{CS}}$ is represented by Equation (2) in order to adjust $L_{\mathrm{CS}}$ to the measuring range of FBRM.

$$
1 \mu \mathrm{m} \leq L_{\mathrm{CS}} \leq 1000 \mu \mathrm{m}
$$

Secondly, projection area $\boldsymbol{P}_{\mathrm{prj}}$ of crystal seen from FBRM probe window is defined from crystal shape $\boldsymbol{P}$ and rotation angles $\theta_{x}, \theta_{y}$, and $\theta_{z}$ around 3 axes. At this time, Equations (3) and (4) are established.

$$
\begin{aligned}
& \boldsymbol{P}_{\mathrm{rot}}\left(\boldsymbol{P}, \theta_{x}, \theta_{y}, \theta_{z}\right) \\
& =\left(\begin{array}{ccc}
\cos \theta_{z} & -\sin \theta_{z} & 0 \\
\sin \theta_{z} & \cos \theta_{z} & 0 \\
0 & 0 & 1
\end{array}\right)\left(\begin{array}{ccc}
\cos \theta_{y} & 0 & \sin \theta_{y} \\
0 & 1 & 0 \\
-\sin \theta_{y} & 0 & \cos \theta_{y}
\end{array}\right)\left(\begin{array}{ccc}
1 & 0 & 0 \\
0 & \cos \theta_{x} & -\sin \theta_{x} \\
0 & \sin \theta_{x} & \cos \theta_{x}
\end{array}\right) \boldsymbol{P} \\
& \boldsymbol{P}_{\mathrm{prj}}\left(\boldsymbol{P}_{\mathrm{rot}}\right)=\left(\begin{array}{lll}
x_{\mathrm{rot}, 1} & x_{\mathrm{rot}, 2} & \ldots \\
y_{\mathrm{rot}, 2} & y_{\mathrm{rot}, 2} & \ldots
\end{array}\right)
\end{aligned}
$$

where the subscript rot means vertex coordinate after rotation, and projection 
area $\boldsymbol{P}_{\mathrm{prj}}$ simply discards information on $z$ axis of $\boldsymbol{P}_{\text {rot }}$. Then, domains of $\theta_{x}$, $\theta_{y}$, and $\theta_{z}$ are represented by Equation (5).

$$
\begin{aligned}
& 0 \leq \theta_{x} \leq 2 \pi \\
& 0 \leq \theta_{y} \leq 2 \pi \\
& 0 \leq \theta_{z} \leq 2 \pi
\end{aligned}
$$

Thirdly, because the beam scans crystals along the $z x$ plane, translation toward $z$ axis or $x$ axis direction doesn't change the relationship between projection area and trajectory of the beam. Therefore, projection area $\boldsymbol{P}_{\text {transl }}$ including information on the distance from trajectory of the beam is defined only from $\boldsymbol{P}_{\text {prj }}$ and translation $y_{d}$ toward $y$ axis. At this time, Equation (6) is established.

$$
\boldsymbol{P}_{\text {transl }}\left(\boldsymbol{P}_{\mathrm{prj}}, y_{d}\right)=\left(\begin{array}{ccc}
x_{\mathrm{rot}, 1} & x_{\mathrm{rot}, 2} & \cdots \\
y_{\mathrm{rot}, 1}+y_{d} & y_{\mathrm{rot}, 2}+y_{d} & \cdots
\end{array}\right)
$$

where the subscript transl means vertex coordinate after translation, and $\boldsymbol{P}_{\text {transl }}$ is the matrix which adds $y_{d}$ to $y$ coordinates of vertex coordinates $\boldsymbol{P}_{\mathrm{prj}}$ before translation. Then, a domain of $y_{d}$ is represented by Equation (7).

$$
-y_{d, \max } \leq y_{d} \leq y_{d, \max }
$$

where it is desirable that $y_{d, \max }$ is large enough for the largest crystal to be calculated. In this paper, $y_{d, \max }$ is defined as the distance between 2 points which are the most distant from each other of all the vertex coordinates of the crystal the characteristic crystal size of which is $1000 \mu \mathrm{m}$.

Lastly, chord length $L_{\mathrm{CL}}$ is defined as the length of line intersection of projection area $\boldsymbol{P}_{\text {transl }}$ and an $x$ axis. To calculate $L_{\mathrm{CL}}$ in a uniform manner, intersections $x_{p, k}$ of an $x$ axis and line segments between all combinations of 2 points from $m$ vertex coordinates of $\boldsymbol{P}_{\text {transl }}$ are to be calculated. In the case that the line segment and an $x$ axis correspond and that the line segment and an $x$ axis don't intersect, $x_{p, k}$ is defined as not a number $(\mathrm{NaN})$. At this time, Equation (8) is established.

$$
x_{p, k}\left(\boldsymbol{P}_{\text {transl }}\right)=\left\{\begin{array}{l}
\operatorname{NaN}\left(y_{\text {transl }, i} y_{\text {transl }, j}>0 \text { or } y_{\text {transl }, i}=y_{\text {transl }, j}=0\right) \\
x_{\text {transl }, i}-\frac{x_{\text {transl }, j}-x_{\text {transl }, i}}{y_{\text {transl }, j}-y_{\text {transl }, i}} y_{\text {transl }, i}(\text { the other })
\end{array}\right.
$$

The set of ${ }_{m} C_{2}$ intersection coordinates $x_{p, k}$ is represented by a vector $\boldsymbol{X}_{p}$ in Equation (9).

$$
\boldsymbol{X}_{p}=\left(\begin{array}{lllll}
x_{p, 1} & \cdots & x_{p, k} & \cdots & x_{p, m} C_{2}
\end{array}\right)
$$

At this time, the chord length $L_{C L}$ is represented by Equation (10).

$$
L_{C L}\left(\boldsymbol{X}_{p}\right)=\left\{\begin{array}{l}
\max \left(\boldsymbol{X}_{p}\right)-\min \left(\boldsymbol{X}_{p}\right)\left(\max \left(\boldsymbol{X}_{p}\right)-\min \left(\boldsymbol{X}_{p}\right) \geq 1 \mu \mathrm{m}\right) \\
0\left(\max \left(\boldsymbol{X}_{p}\right)-\min \left(\boldsymbol{X}_{p}\right)<1 \mu \mathrm{m} \text { or } \max \left(\boldsymbol{X}_{p}\right)-\min \left(\boldsymbol{X}_{p}\right)=\mathrm{NaN}\right)
\end{array}\right.
$$

However, when $L_{\mathrm{CL}}$ is smaller than the measuring lower limit or all of the in- 
tersection coordinates $x_{p, k}$ are $\mathrm{NaN}, L_{\mathrm{CL}}$ is defined as 0 , and the range of $L_{\mathrm{CL}}$ is represented by Equation (11).

$$
L_{\mathrm{CL}}=0 \text { or } 1 \mu \mathrm{m} \leq L_{\mathrm{CL}} \leq 1000 \mu \mathrm{m}
$$

\subsection{Strict Transformation from CSD to CLD with Multiple Integral}

A domain of crystal size in the th fraction when the domain of crystal size in Equation (2) is divided into $n$ equal parts by a logarithmic scale is represented by Equation (12).

$$
L_{\mathrm{CS}, \mathrm{j}} \leq L_{\mathrm{CS}} \leq L_{\mathrm{CS}, j+1}, L_{\mathrm{CS}, j}=10^{\frac{3}{n}(j-1)} \mu \mathrm{m}
$$

Similarly, a range of chord length in the th fraction when the range of chord length in Equation (11) except 0 is divided into $n$ equal parts by a logarithmic scale is represented by Equation (13).

$$
L_{\mathrm{CL}, i} \leq L_{\mathrm{CL}} \leq L_{\mathrm{CL}, i+1}, L_{\mathrm{CL}, i}=10^{\frac{3}{n}(i-1)} \mu \mathrm{m}
$$

Originally, it is not necessarily required that the number of fractions on crystal size is the same to that on chord length. At this time, the probability that one of an infinitely large number of crystals in Equation (12) is measured as the chord length in Equation (13) is to be calculated. First of all, $L_{\mathrm{CS}}$ by a logarithmic scale and $\theta_{x}, \theta_{y}, \theta_{z}$, and $y_{d}$ by a linear scale are assumed to be distributed uniformly in Equation (12), (5), and (7) respectively. Therefore, joint probability density function $f$ of 5 independent variables satisfies the relationship expressed by Equation (14).

$$
\frac{\partial f}{\partial\left(\log L_{\mathrm{CS}}\right)}=\frac{\partial f}{\partial \theta_{x}}=\frac{\partial f}{\partial \theta_{y}}=\frac{\partial f}{\partial \theta_{z}}=\frac{\partial f}{\partial y_{d}}=0
$$

Then, 5 variables are arranged to be denoted by a vector $s$ in Equation (15).

$$
\mathrm{d} \boldsymbol{s}=\mathrm{d}\left(\log L_{\mathrm{CS}}\right) \mathrm{d} \theta_{x} \mathrm{~d} \theta_{y} \mathrm{~d} \theta_{z} \mathrm{~d} y_{d}
$$

Moreover, domains of 5 variables in Equations (12), (5), and (7) are arranged to be denoted by $E_{j} \subset \mathbb{R}^{5}$, and Equation (16) is established.

$$
K_{j}=K=\int_{E_{j}} \mathrm{~d} s
$$

where the integrated value in all of the domains $K$ is independent of fraction number $j$ because the integrated values in all of the fractions by a logarithmic scale are the same to one another when the domain of crystal size is divided into equal parts by a logarithmic scale. In the domains $E_{j}$, the probability $(S)_{i j}$ that a crystal is measured as the chord length in Equation (13) is represented by Equation (17).

$$
(S)_{i j}=\frac{\int_{s \in E_{j}}}{\substack{L_{\mathrm{CL}, i} \leq L_{\mathrm{CL}} \leq L_{\mathrm{CL}, i+1} \\ K_{j}}}=\frac{M_{i j}}{K} \propto M_{i j}
$$

Because chord length is clearly defined from 5 independent variables (see Section 2.2), the integrated value $M_{i j}$ in the target range is uniquely calculated for 
each combination of fraction numbers $i$ and $j$. In addition, $M_{i j}$ doesn't change with $y_{d, \max }$ when $y_{d, \max }$ is larger than a certain value (see Section 2.2). In actual vessels, $y_{d, \max }$ is much larger than this value and so the value of $M_{i j}$ is the same to the value calculated in Equation (17). Therefore, if Equation (17) is computable, the probability is strictly calculated. This probability is a contribution of the domain of crystal size in the th fraction to the range of chord length in the ith fraction. Contributions of the domains of crystal size in $n$ fractions are multiplied by the numbers of crystals and summed up to calculate the expected value of the range of chord length in the ith fraction. Therefore, Equation (18) is established.

$$
N_{\mathrm{CLD}, i}=\sum_{j=1}^{n}(\boldsymbol{S})_{i j} N_{\mathrm{CSD}, j}
$$

where $N_{\text {CLD }, i}$ is the count of crystals with chord lengths in the th fraction and $N_{\text {CSD }, j}$ is the number of crystals with crystal sizes in the $t$ th fraction. Equation (18) can be generalized and by using vectors $N_{\text {CLD }}$ and $N_{\text {CSD }}$ which adapt vector indices to fraction numbers of CLD and CSD, Equation (19) is established.

$$
\boldsymbol{N}_{\mathrm{CLD}}=\boldsymbol{S} \boldsymbol{N}_{\mathrm{CSD}}
$$

For the following discussion, $S$ is called shape transformation matrix.

\subsection{Approximate Transformation from CSD to CLD with Monte Carlo Analysis}

The integration range of Equation (17) is too complex for the exact solution to be obtained. Therefore, Monte Carlo analysis is performed with uniformly distributed pseudorandom number $r$. First of all, $r$ is uniformly distributed in the range represented by Equation (20).

$$
0<r<1
$$

At this time, to make 5 independent variables have the domains in Equations (12), (5), and (7), and to make Equation (14) about probability density established, 5 independent variables are defined as Equations (21), (22), and (23) with random number.

$$
\begin{gathered}
\log L_{\mathrm{CS}}=\log L_{\mathrm{CS}, j}+\left(\log L_{\mathrm{CS}, j+1}-\log L_{\mathrm{CS}, j}\right) r_{1} \Leftrightarrow L_{\mathrm{CS}}=L_{\mathrm{CS}, j}\left(\frac{L_{\mathrm{CS}, j+1}}{L_{\mathrm{CS}, j}}\right)^{r_{1}} \\
\theta_{x}=2 \pi r_{2} \\
\theta_{y}=2 \pi r_{3} \\
\theta_{z}=2 \pi r_{4} \\
y_{d}=y_{d, \text { max }}\left(2 r_{5}-1\right)
\end{gathered}
$$

However, Equation (22) is established only if the directions of crystals are uniformly distributed regardless of crystal shape and the direction of the suspension flow. 5 random numbers change for each trial and the dependent variable $L_{\mathrm{CL}}$ is calculated on each trial. By using the total number of trials $K_{\mathrm{MC}}$ instead of 
sample space $K$ in Equation (17) and the number of times $M_{\mathrm{MC}, i j}$ that the events have happened (i.e. the number of times that crystals have measured as the chord length in Equation (13)) instead of probability event $M_{i j}$, the probability is calculated likewise in Equation (17) to obtain Equation (24).

$$
\frac{M_{\mathrm{MC}, i j}}{K_{\mathrm{MC}}}=\left(\boldsymbol{S}_{\mathrm{MC}}\right)_{i j} \stackrel{K_{\mathrm{MC}} \rightarrow \infty}{\longrightarrow}(S)_{i j}
$$

where the subscript MC means the value about Monte Carlo analysis. If $r$ is true random number, the approximate probability $\left(S_{\mathrm{MC}}\right)_{i j}$ approaches the exact probability $(S)_{i j}$ as the total number of trials $K_{\mathrm{MC}}$ increases. In this paper, pseudorandom number was created with MATLAB 7.5.0 (R2007b).

\section{Experiments}

\subsection{Substances}

In this paper, the verification experiment was performed with acetaminophen $\left(\mathrm{CH}_{3} \mathrm{CONHC}_{6} \mathrm{H}_{4} \mathrm{OH}\right.$, abbreviated to AAP) and L-arginine $\left(\mathrm{C}_{6} \mathrm{H}_{14} \mathrm{~N}_{4} \mathrm{O}_{2}\right.$, abbreviated to Arg). AAP, the molecular weight of which is 151.16 , is a white crystalline compound, hardly soluble in water and readily soluble in ethanol. AAP has 3 kinds of polymorphs. AAP is often used as an analgesic antipyretic or a cold medicine. In the verification experiment, ethanol was purchased from Wako Pure Chemical Industries, Ltd. (Osaka, Japan) and AAP from Tokyo Chemical Industry Co., Ltd. (Tokyo, Japan). Then, Arg, the molecular weight of which is 174.02 , is a white crystalline basic amino acid, readily soluble in water and hardly soluble in ethanol. Arg has 2 kinds of pseudo polymorphs: anhydrate and dehydrate. Arg also activates immune function and accelerates cell proliferation. In the verification experiment, Arg was purchased from Wako Pure Chemical Industries, Ltd. (Osaka, Japan).

\subsection{Experimental Apparatus}

Solution temperature and CLD were measured with the apparatus shown in Figure 2.

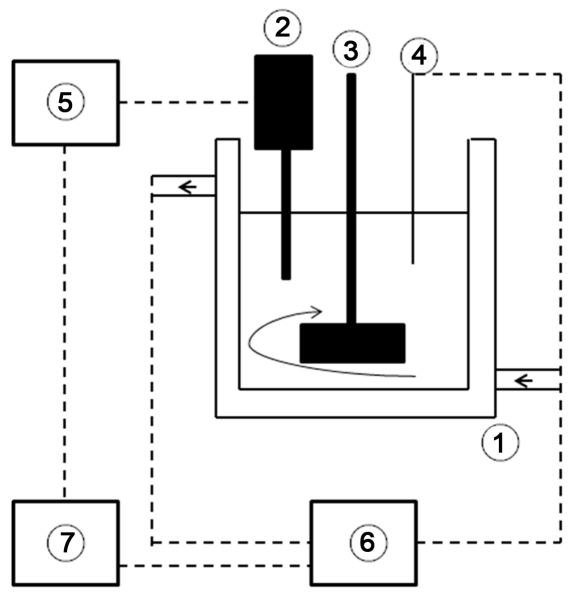

1) $500 \mathrm{~mL}$ jacketed glass vessel (2) FBRM probe

(3) 4-Paddle agitator

(4) Temperature probe

(5) FBRM main body

6) Temperature control system

(7) Computer

Figure 2. Experimental apparatus 
Solution temperature was measured with platinum electrode ( $\mathrm{Pt100,}$ JISC1604-1997/IEC 751). CLD was measured with FBRM (made by Mettler-Toledo, model G400).

Measurement conditions of FBRM are described below.

- The measuring range is $1-1000 \mu \mathrm{m}$.

- The measurement mode is Macro.

- The measuring range is divided into 30 equal parts by a logarithmic scale.

- The wavelength of the laser beam is $780 \mu \mathrm{m}$.

\subsection{Experimental Procedure}

\subsubsection{Verification Experiment with AAP}

AAP (45 g) was added to ethanol $(300 \mathrm{~mL})$ to prepare a saturated solution at $20^{\circ} \mathrm{C}$. Then, with the solution held at $20^{\circ} \mathrm{C}$, AAP seed crystals were added to the solution under 5 conditions. The suspension of AAP in ethanol was stirred and the crystals were washed for about 30 min with CLD from FBRM measured. After it was confirmed that CLD was steady, the suspension was sampled at the same time that CLD was recorded and CSD was measured with an optical microscope. The experimental condition is showed in Table 1.

In Table 1, coarse seed means crystals from a reagent bottle and fine seed means crystals crashed with a mortar.

\subsubsection{Verification Experiment with Arg}

The experiment with suspension of Arg in water was performed likewise in section 3.3.1. The experimental condition is showed in Table 1.

\subsubsection{Creation of Shape Transformation Matrix}

Shape transformation matrix $S$ on each substance was created with MATLAB 7.5.0 (R2007b). At this time, AAP crystal images (Figure 3) obtained in section 3.3.1 and Arg crystal images (Figure 4) obtained in section 3.3.2 were used as

Table 1. Experimental condition.

\begin{tabular}{|c|c|c|c|c|c|c|c|}
\hline $\begin{array}{l}\text { Cond. } \\
\text { No. }\end{array}$ & Substance & $\begin{array}{c}\text { Mass of } \\
\text { solute } \\
{[\mathrm{kg}]}\end{array}$ & $\begin{array}{c}\text { Mass of } \\
\text { solvent } \\
{[\mathrm{kg}]}\end{array}$ & $\begin{array}{c}\text { Agitation } \\
\text { rate } \\
{[\mathrm{rpm}]}\end{array}$ & $\begin{array}{c}\text { Saturation } \\
\text { temperature } \\
{\left[{ }^{\circ} \mathrm{C}\right]}\end{array}$ & $\begin{array}{c}\text { Mass of } \\
\text { fine seed } \\
{[\mathrm{g}]}\end{array}$ & $\begin{array}{c}\text { Mass of } \\
\text { coarse seed } \\
{[\mathrm{g}]}\end{array}$ \\
\hline 1 & & & & & & 2 & 2 \\
\hline 2 & & & & & & 4 & 2 \\
\hline 3 & AAP & 0.045 & 0.237 & 300 & 20 & 6 & 2 \\
\hline 4 & & & & & & 2 & 4 \\
\hline 5 & & & & & & 2 & 6 \\
\hline 6 & & & & & & 2 & 2 \\
\hline 7 & & & & & & 4 & 2 \\
\hline 8 & Arg & 0.048 & 0.3 & 300 & 20 & 6 & 2 \\
\hline 9 & & & & & & 2 & 6 \\
\hline 10 & & & & & & 2 & 8 \\
\hline
\end{tabular}




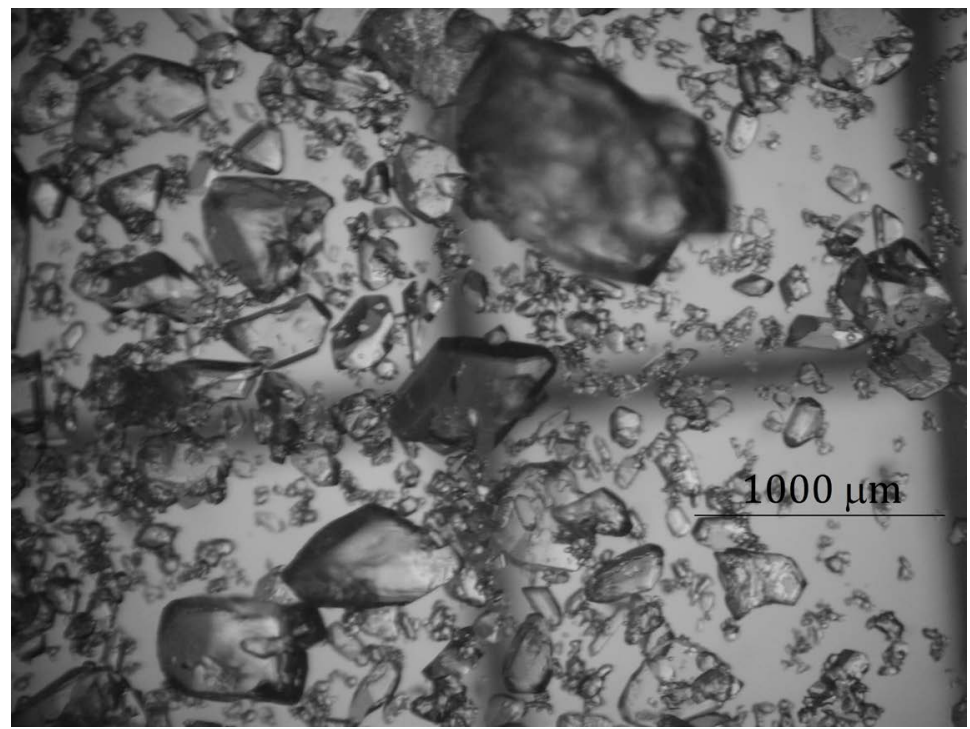

Figure 3. Sample of AAP crystal image. Saturation was set to -100 with Microsoft Office 2010. Scale bar the length of which was calculated from a micrometer was inserted with Microsoft Paint.

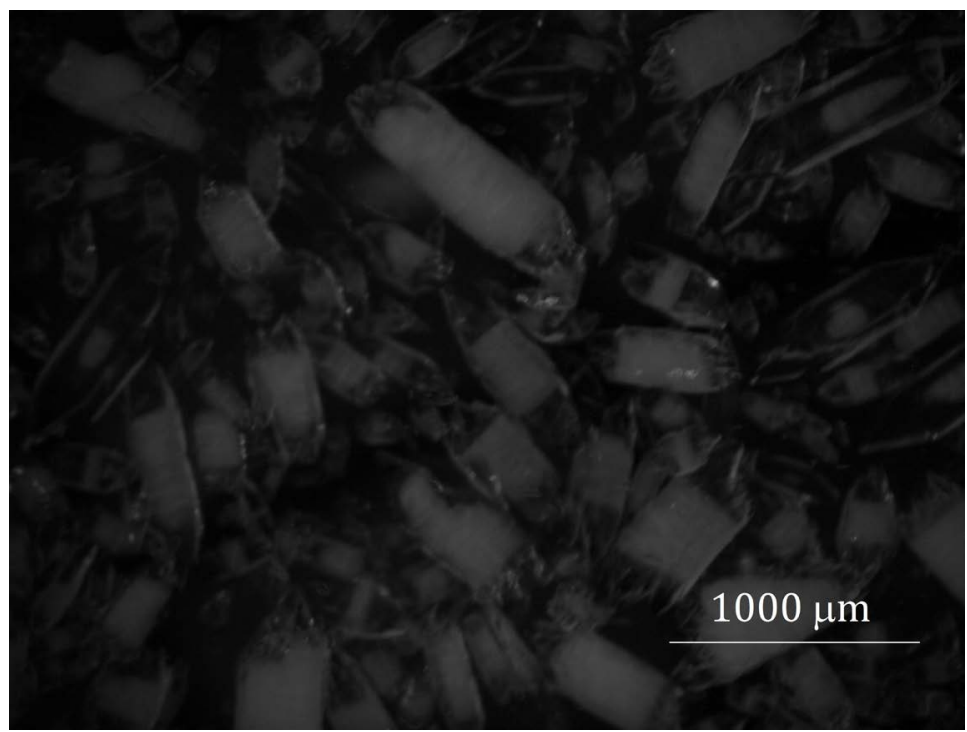

Figure 4. Sample of Arg image. Saturation was set to -100 and brightness to 100 with Microsoft Office 2010. Scale bar the length of which was calculated from a micrometer was inserted with Microsoft Paint.

reference, and crystal shape $\boldsymbol{P}\left(L_{\mathrm{CS}}\right)$ of each crystal was defined as following. The crystal shape of AAP is similar regardless of crystal size and the shape is an octahedron of 3 axes ratio of 1:1:1.5 which intersect at the middle points (Figure 5). The crystal shape of Arg is similar regardless of crystal size and the shape is a rectangular solid of 3 sides ratio of 1:1:3 (Figure 6).

In addition, the characteristic crystal size of each substance was defined as the black line of each model shape in Figure 5 and Figure 6 . The total number of trials in Monte Carlo analysis was 25,000,000 and the size of shape transformation matrix was $30 \times 30$. 


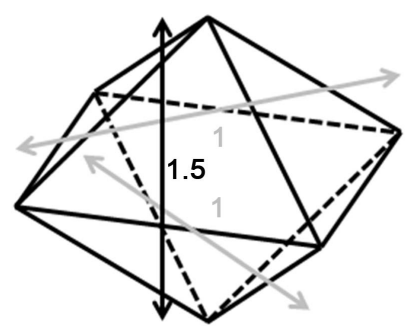

Figure 5. Model shape of AAP.

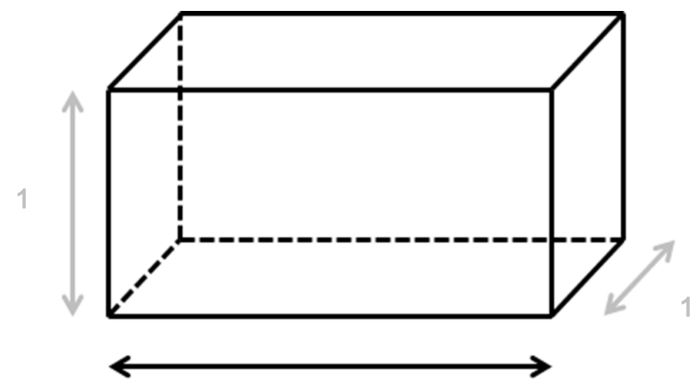

3

Figure 6. Model shape of Arg.

\subsection{Error Evaluation}

The absolute value of CLD hardly has quantitative information, because $y_{d, \max }$ in an actual system is unknown and a much larger value than was used in probability calculation, and changes with time. However, when $y_{d, \max }$ is sufficiently large, each relative value of elements contained in $S$ doesn't change with $y_{d, \max }$. Therefore, if CSD after shape transformation is directly proportional to CLD, it can be confirmed that the shape transformation matrix is accurate and that the theory in this paper is valid. In addition, in the case that CSD tries to be measured with FBRM apparatus in practice, CSD cannot be calculated with CLD and $S$ immediately from the above reason. At this time, the data needs handling correctly. For example, the concentration is measured secondarily and temporary CSD is multiplied by a constant based on mass balance. In this case, $L^{3}$-weighted distribution is usually used, and so the shape transformation matrix $S$ is assumed to function the best when the error of $L^{3}$-weighted distribution is practically small. $L^{3}$-Weighted distribution $\boldsymbol{\mu}_{3}$ is calculated from no-weighted distribution $\boldsymbol{N}$ by using Equation (25).

$$
\boldsymbol{\mu}_{3}=\boldsymbol{L}^{3} \boldsymbol{N}, \boldsymbol{L}=\left[\begin{array}{cccccc}
L_{R 1} & 0 & \cdots & 0 & \cdots & 0 \\
0 & L_{R 2} & \cdots & 0 & \cdots & 0 \\
\vdots & \vdots & \ddots & \vdots & \ddots & \vdots \\
0 & 0 & \cdots & L_{R i} & \cdots & 0 \\
\vdots & \vdots & \ddots & \vdots & \ddots & \vdots \\
0 & 0 & \cdots & 0 & \cdots & L_{R n}
\end{array}\right], L_{R i}=\sqrt{L_{i} L_{i+1}}
$$

where $L$ is a diagonal matrix the th diagonal element of which is the average of the $i$ th fraction $L_{R i}$ and $L_{R i}$ is the geometric average of both ends $L_{i}$ and $L_{i+1}$ of the $i$ th fraction. For 3 no-weighted distributions: CSD measured with an 
optical microscope before and after the transformation and CLD from FBRM, $L^{3}$-weighted distributions were calculated by using Equation (25). Then, every $L^{3}$-weighted distribution was normalized and the total amount of every $L^{3}$-weighted distribution was adjusted to 1 to exclude quantitative discussion. Normalized $L^{3}$-weighted distribution $\boldsymbol{a}$ is represented by Equation (26).

$$
\boldsymbol{a}=\frac{\boldsymbol{\mu}_{3}}{\left\|\boldsymbol{\mu}_{3}\right\|_{1}}
$$

At this time, CSD measured with an optical microscope was assumed to be a calculated vector, CLD from FBRM a observed vector, and relative error $E$ was defined as a 2-norm of difference between a calculated vector and observed one. $E$ is represented by Equation (27).

$$
E=\left\|\boldsymbol{a}_{\text {calc }}-\boldsymbol{a}_{\text {obs }}\right\|_{2}
$$

$E$ from CSD after the transformation was compared with that before the transformation, and the validity of $S$ was discussed.

\subsection{Test of Properties of Matrices with Model CSD}

The properties of the shape transformation matrices of AAP and Arg, which were created in section 3.3.3 and the validity of which was demonstrated in section 3.4, were tested by being multiplied by the following 2 CSD models to demonstrate the utility of the transformation. Figure 7 shows the case that crystals exist only in fraction No. 28 and Figure 8 shows the case that every fraction has the same number of crystals. The total amount of every CSD model is adjusted to 1 .

\section{Results and Discussion}

For example, normalized $L^{3}$-weighted distributions under cond. 3 and cond. 9 are shown in Figure 9 and Figure 10 respectively.

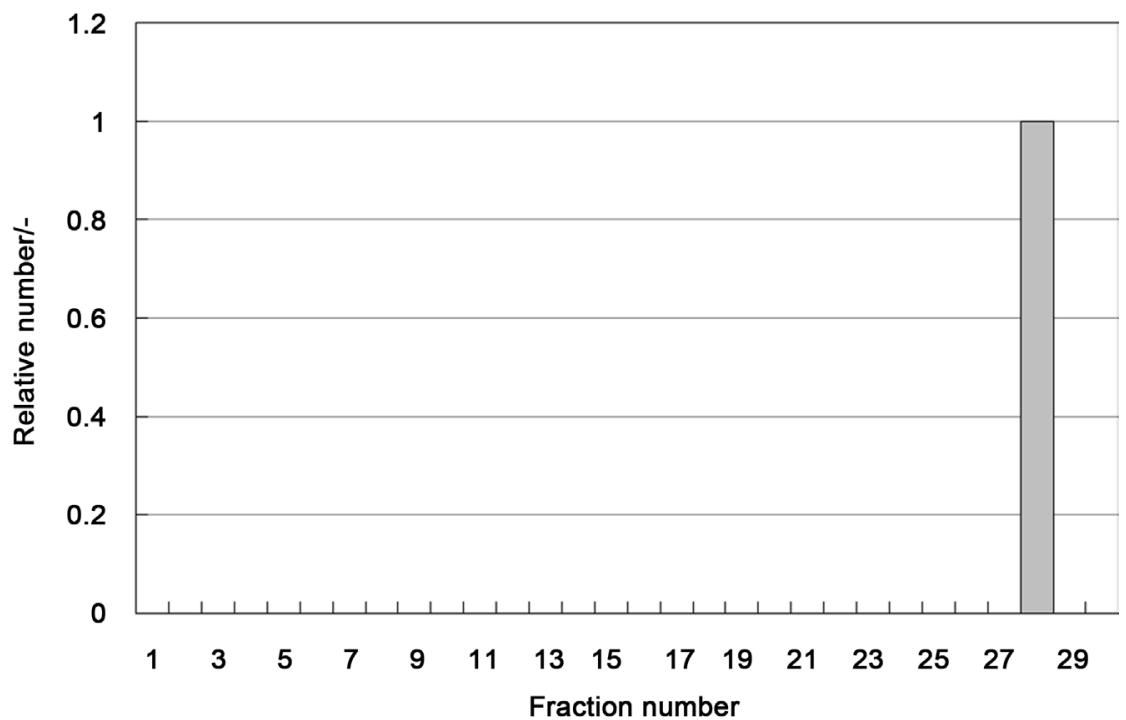

Figure 7. CSD model 1. 


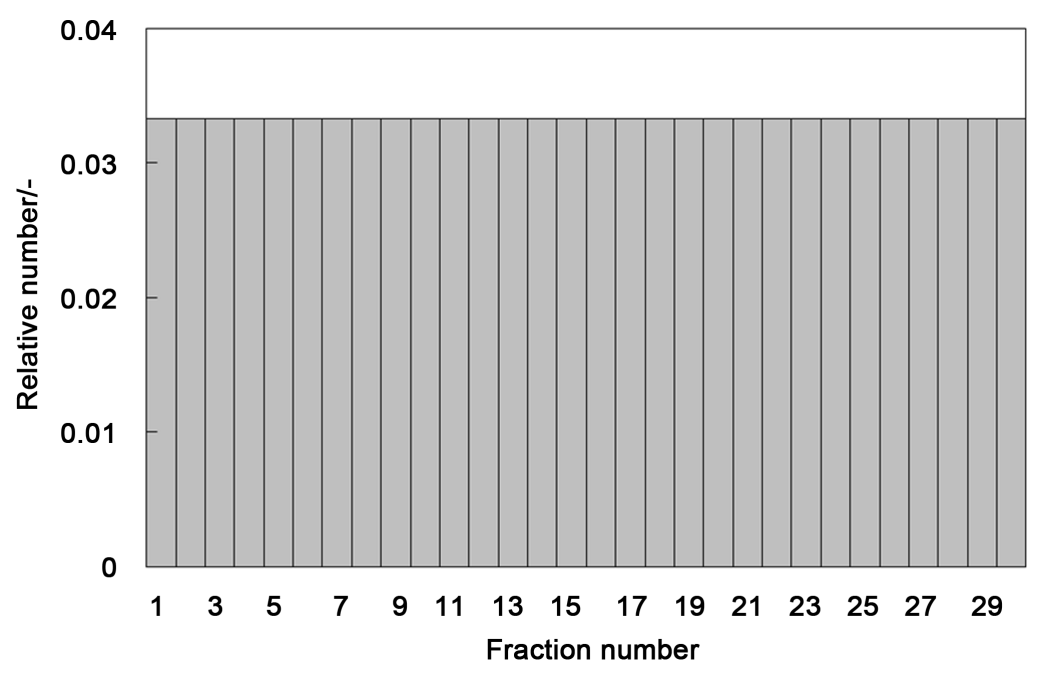

Figure 8. CSD model 2.

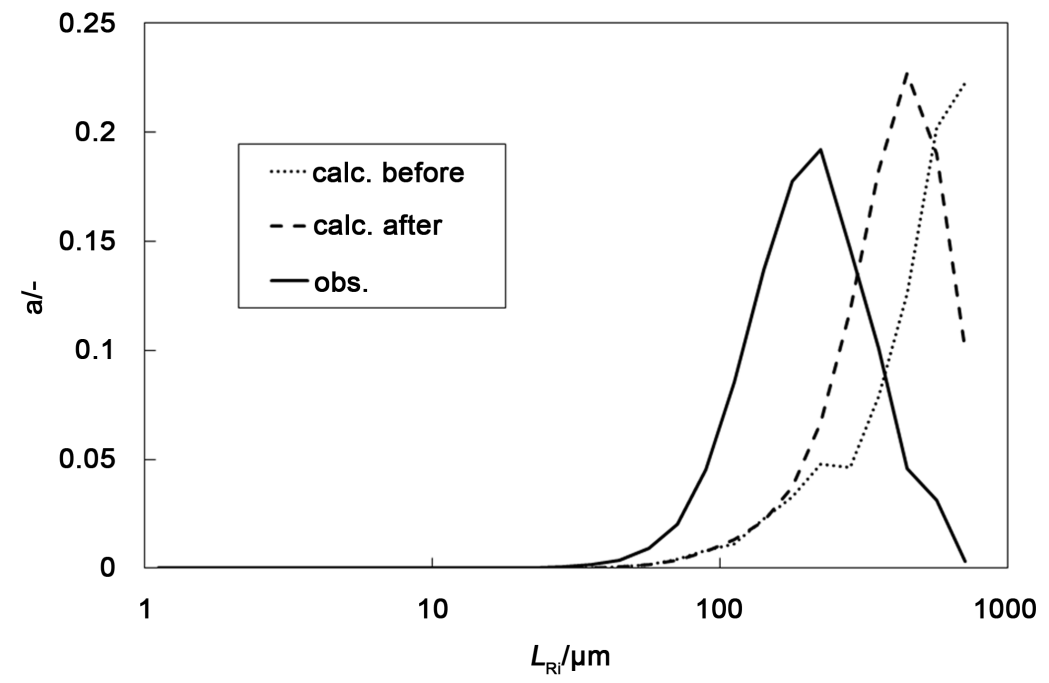

Figure 9. Normalized $L^{3}$-weighted distribution under cond. 3.

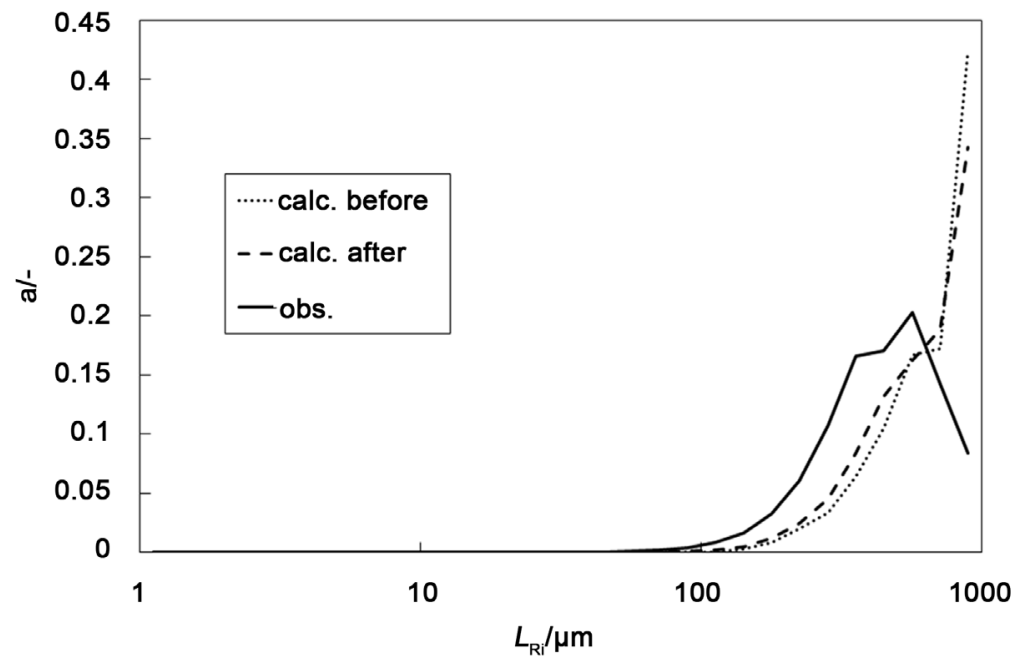

Figure 10. Normalized $L^{3}$-weighted distribution under cond. 9. 
In Figure 9 and Figure 10, calc. means values from optical microscopy, obs. values from FBRM, and before and after values before and after the shape transformation respectively. The examples in Figure 9 and Figure 10 show that CSD was transformed to approach CLD in both systems. The results of the error evaluation performed in the manner of section 3.4 are shown in Table 2.

At this time, the rate of change in relative error obtained before and after shape transformation was calculated. In addition, the average of the rate of change by each substance was calculated to demonstrate the validity of the shape transformation matrix on each substance.

Table 2 shows that contrary to expectations the error was increased by the transformation under cond. 7 and cond. 8. This phenomenon was assumed to occur because under these 2 conditions the suspensions contained many fine seed crystals, the aspect ratio of which was smaller than that of model shape defined in Figure 6. Therefore, this result shows that $S$ didn't function correctly when actual crystal shape differed greatly from defined crystal shape $\boldsymbol{P}$. However, the errors under the other conditions and the average by each substance show that the errors were almost always decreased greatly and that the method in this paper was assumed to be valid.

Then, CLDs which $S$ on AAP and Arg, the validity of which had been demonstrated, multiplied by 2 CSD models shown in Figure 7 and Figure 8 became are shown in Figures 11-14. The total amount of every CLD was adjusted to 1 .

Figure 11 and Figure 12 show that both of the distributions were assumed to become broad in contrast to the monodispersed system in Figure 7 and that both of the most frequent values came to exist in fraction No. 25 less than the fraction number in which the most frequent value of CSD model 1 had existed by 3. This phenomenon was assumed to show that crystals were rarely measured as the same chord length as the characteristic crystal size and that almost all of the crystals were measured around the edge or at a slant. In addition, crystals

Table 2. Error evaluation.

\begin{tabular}{cccccccc}
\hline Cond. No. & Substance & $\begin{array}{c}\text { Mass of } \\
\text { fine seed }[\mathrm{g}]\end{array}$ & $\begin{array}{c}\text { Mass of } \\
\text { coarse seed }[\mathrm{g}]\end{array}$ & $\begin{array}{c}E_{\text {before }}[-] \\
E_{\text {after }} \\
{[-]}\end{array}$ & $\begin{array}{c}\text { Changing } \\
\text { rate }[\%]\end{array}$ & $\begin{array}{c}\text { Ave. } \\
{[\%]}\end{array}$ \\
\hline 1 & 2 & 2 & 0.731 & 0.494 & -32.41 & \\
2 & & 4 & 2 & 0.615 & 0.464 & -24.45 & \\
3 & AAP & 6 & 2 & 0.440 & 0.362 & -17.77 & -24.46 \\
4 & & 2 & 4 & 0.681 & 0.491 & -27.83 & \\
5 & & 2 & 6 & 0.577 & 0.463 & -19.86 & \\
6 & & 2 & 2 & 0.126 & 0.124 & -1.52 & \\
7 & & 4 & 2 & 0.208 & 0.212 & 2.01 & \\
8 & \multirow{2}{*}{ Arg } & 6 & 2 & 0.168 & 0.205 & 22.28 & -4.22 \\
9 & & 2 & 6 & 0.375 & 0.292 & -22.71 & \\
10 & & 2 & 8 & 0.232 & 0.182 & -21.71 & \\
\hline
\end{tabular}




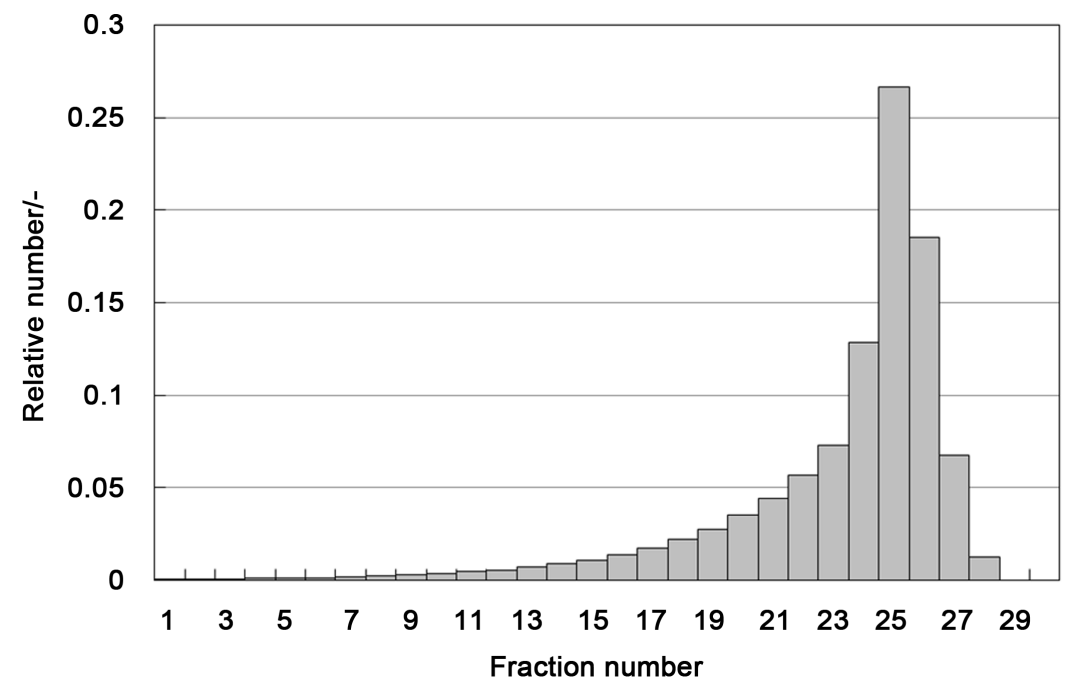

Figure 11. AAP CLD from CSD model 1.

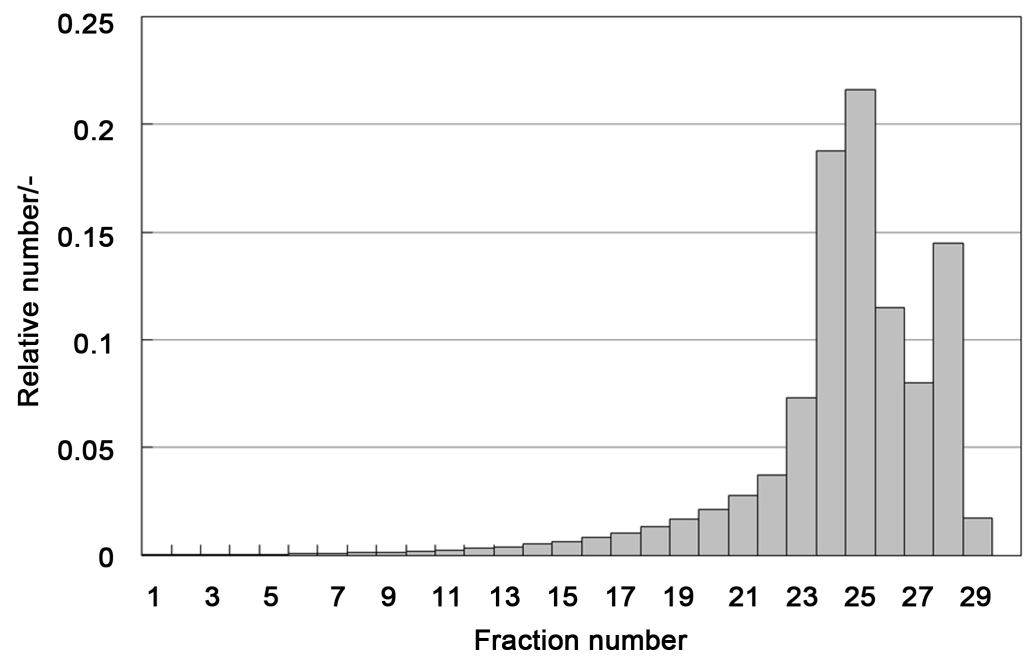

Figure 12. Arg CLD from CSD model 1.

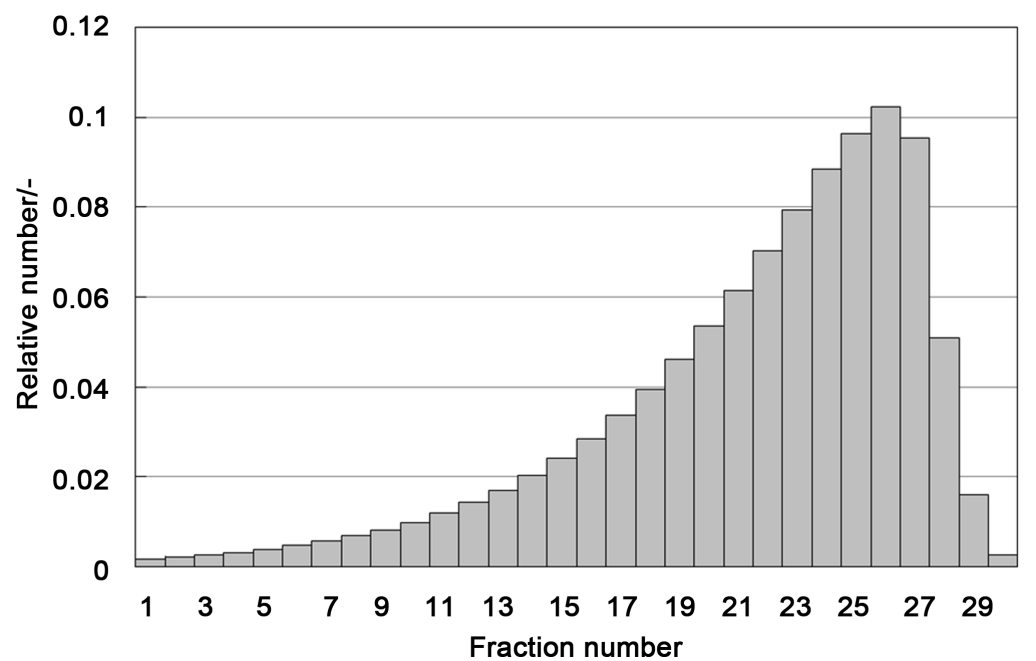

Figure 13. AAP CLD from CSD model 2. 


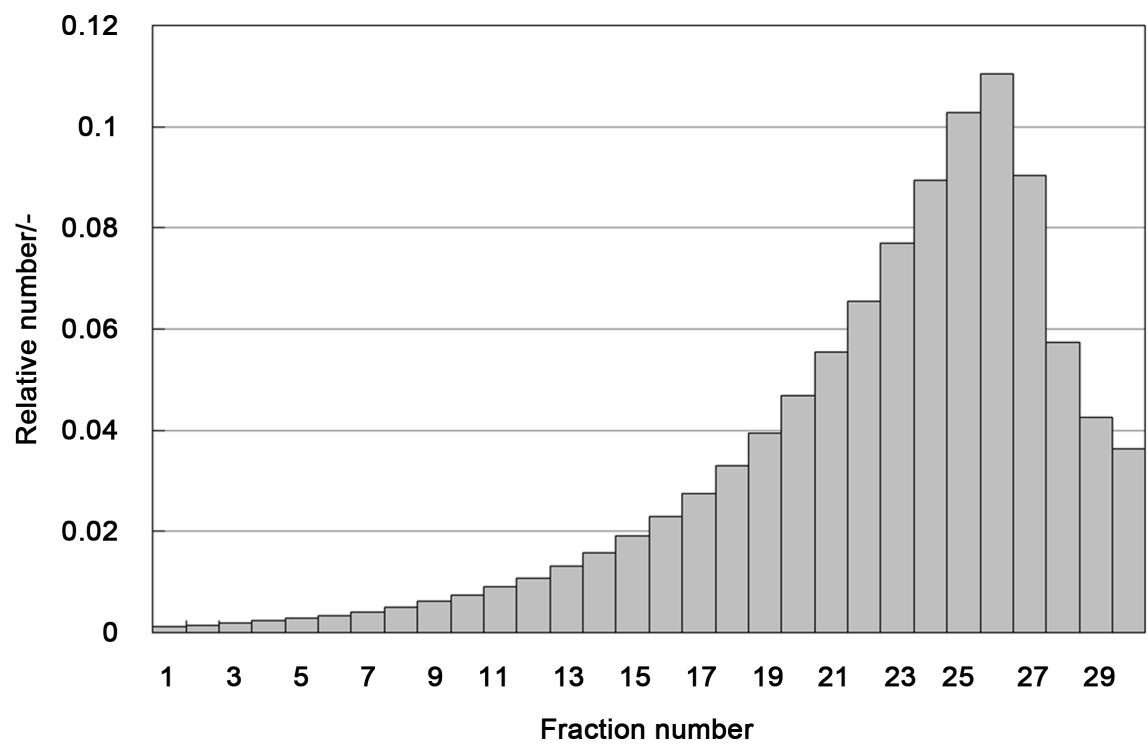

Figure 14. Arg CLD from CSD model 2.

were sometimes measured as a longer chord length than the characteristic crystal size only for Arg. This was because the chord length around the body diagonal line was longer than the characteristic crystal size. The effect that crystals were measured around the edge or at a slant affected CSD complexly, depending on crystal shape. For example, Figure 12 shows that CLD was split in contrast to the monodispersed system in Figure 7. Inversely, CSD doesn't necessarily show multiple peaks when CLD shows multiple peaks, which is assumed to show that serious errors can occur in the case that CLD is handled as it is as CSD. Moreover, taking the fact that CLD wasn't split in Figure 11 into account, it is assumed that this effect heavily depends on crystal shape and that the data cannot be transformed in a unified manner for all of the crystal shapes.

Then, Figure 13 and Figure 14 show that the relative number was increased as the chord length became larger within the ranges of small fraction numbers and of middle fraction numbers in contrast to the uniform distribution in Figure 8. This was because the probability that the crystals overlapped with trajectory of the beam was decreased as the crystal size became smaller against the fixed constant $y_{d \cdot}$. However, within the ranges of large fraction numbers, the trend was reversed. This was assumed to occur because of the effect that crystals were measured around the edge or at a slant, which is mentioned above.

Consequently, the state of CSD cannot be discussed from CLD without using shape transformation matrix, and the utility of the shape transformation matrix calculated in this paper was assumed to be demonstrated.

\section{Conclusions}

By using Monte Carlo analysis, shape transformation matrices which transformed CSD into CLD for the crystal shape defined beforehand were created. The validity of these shape transformation matrices were tested with the suspension of AAP in ethanol and the suspension of Arg in water. The verification ex- 
periments show that the relative error between CLD and CSD after transformation was significantly smaller than that between CLD and CSD before transformation only in the case that the actual crystal shapes corresponded with the definition. Therefore, the validity of this transformation method of CSD with the shape transformation matrix was demonstrated. Then, the virtual experiments in which the CLDs were obtained by the shape transformation matrices multiplied by some CSD models show that the trend and the statistics of CSD greatly differed from those of CLD and that the degree of the difference depended on the crystal shape. In other words, the state of CSD cannot be discussed from CLD without using shape transformation matrix, and the utility of the shape transformation matrix calculated in this paper was demonstrated.

In this paper, the crystal shape was assumed to be similar regardless of crystal size for simplicity, but actually, shape transformation matrix can be created even if crystal shape is defined as a mapping of the crystal size. This mapping is accurately researched beforehand and inserted in the shape transformation matrix to enable the matrix to shape-transform for more general cases. In addition, by using the shape transformation matrix with the method in this paper for inverse transformation, the algorithm transforming CLD into CSD is created to realize real-time monitoring of CSD with FBRM. Many of the operations containing matrix can be performed in a very short time with numerical analysis software. In other words, the fact that shape-transforming operator was obtained as matrix in this paper seems to contribute to transforming CLD into CSD with the quality of in-line in FBRM remaining.

\section{Acknowledgements}

We express thanks to Mettler-Toledo K.K. BU AutoChem for technical support.

\section{References}

[1] Hanaki, K. and Hirasawa, I. (2009) Observation of Secondary Nucleation in Drowning-Out Crystallization Using FBRM. Journal of Chemical Engineering of Japan, 42, 386-392. https://doi.org/10.1252/jcej.08we198

[2] Ruf, A., Worlitschek, J. and Mazzotti, M. (2000) Modeling and Experimental Analysis of PSD Measurements through FBRM. Particle \& Particle Systems Characterization, 17, 167-179. https://doi.org/10.1002/1521-4117(200012)17:4<167::AID-PPSC167>3.0.CO;2-T

[3] Worlitschek, J., Hocker, T. and Mazzotti, M. (2005) Restoration of PSD from Chord Length Distribution Data Using the Method of Projections onto Convex Sets. Particle \& Particle Systems Characterization, 22, 81-98. https://doi.org/10.1002/ppsc.200400872

[4] Kail, N., Briesen, H. and Marquardt, W. (2007) Advanced Geometrical Modeling of Focused Beam Reflectance Measurements (FBRM). Particle \& Particle Systems Characterization, 24, 184-192. https://doi.org/10.1002/ppsc.200601036 


\section{Nomenclature}

\begin{tabular}{|c|c|c|}
\hline$A$ & normalized $\boldsymbol{\mu}_{3}$ & {$[-]$} \\
\hline$E$ & domain of $s$ & {$\left[\mathrm{rad}^{3} \cdot \mathrm{m}\right]$} \\
\hline$E$ & relative error & {$[-]$} \\
\hline$f$ & joint probability density function & {$\left[\operatorname{rad}^{-3} \cdot \mathrm{m}^{-1}\right]$} \\
\hline$K$ & sample space & {$\left[\mathrm{rad}^{3} \cdot \mathrm{m}\right]$} \\
\hline $\boldsymbol{L}$ & set of $L_{R}$ in diagonal matrix & {$[\mathrm{m}]$} \\
\hline$L_{\mathrm{CL}}$ & chord length & {$[\mathrm{m}]$} \\
\hline$L_{\mathrm{CS}}$ & crystal size & {$[\mathrm{m}]$} \\
\hline$L_{R}$ & geometric average of both ends of fraction & {$[\mathrm{m}]$} \\
\hline$m$ & number of vertices & {$[-]$} \\
\hline$M$ & probability event & {$\left[\mathrm{rad}^{3} \cdot \mathrm{m}\right]$} \\
\hline$N$ & no-weighted distribution & {$[\#]$} \\
\hline$N$ & number of crystals & {$[\#]$} \\
\hline$N$ & number of fractions & {$[-]$} \\
\hline$p$ & position vector of vertex coordinates & {$[\mathrm{m}]$} \\
\hline$p$ & set of $\boldsymbol{p}$ in matrix & {$[\mathrm{m}]$} \\
\hline$r$ & pseudorandom number & {$[-]$} \\
\hline$s$ & set of 5 independent variables & {$\left[\operatorname{rad}^{3} \cdot \mathrm{m}\right]$} \\
\hline$S$ & shape transformation matrix & {$[-]$} \\
\hline$X$ & $x$-coordinate & {$[\mathrm{m}]$} \\
\hline$x_{p}$ & intersection of $x$-axis and line segment & {$[\mathrm{m}]$} \\
\hline $\boldsymbol{X}_{p}$ & set of $x_{p}$ in vector & {$[\mathrm{m}]$} \\
\hline$Y$ & $y$-coordinate & {$[\mathrm{m}]$} \\
\hline$y_{d}$ & translation toward y-axis & {$[\mathrm{m}]$} \\
\hline$y_{d, \max }$ & required minimax value of $y_{d}$ & {$[\mathrm{~m}]$} \\
\hline$Z$ & z-coordinate & {$[\mathrm{m}]$} \\
\hline $\boldsymbol{\mu}_{3}$ & $L^{3}$-weighted distribution & {$\left[\# \mathrm{~m}^{3}\right]$} \\
\hline$\theta_{x}$ & rotation angle around $x$-axis & {$[\mathrm{rad}]$} \\
\hline$\theta_{y}$ & rotation angle around $y$-axis & {$[\mathrm{rad}]$} \\
\hline$\theta_{z}$ & rotation angle around $\mathrm{z}$-axis & {$[\mathrm{rad}]$} \\
\hline after & after transformation & \\
\hline before & before transformation & \\
\hline calc & calculated value & \\
\hline CLD & chord length distribution & \\
\hline CSD & crystal size distribution & \\
\hline MC & Monte Carlo analysis & \\
\hline obs & observed value & \\
\hline prj & projection & \\
\hline rot & after rotation & \\
\hline transl & after translation & \\
\hline AAP & acetaminophen & \\
\hline Arg & L-arginine & \\
\hline FBRM & focused beam reflectance measurement & \\
\hline
\end{tabular}


Submit or recommend next manuscript to SCIRP and we will provide best service for you:

Accepting pre-submission inquiries through Email, Facebook, LinkedIn, Twitter, etc. A wide selection of journals (inclusive of 9 subjects, more than 200 journals)

Providing 24-hour high-quality service

User-friendly online submission system

Fair and swift peer-review system

Efficient typesetting and proofreading procedure

Display of the result of downloads and visits, as well as the number of cited articles Maximum dissemination of your research work

Submit your manuscript at: http://papersubmission.scirp.org/

Or contact aces@scirp.org 